\title{
BMJ Open Proactive approaches to identifying dementia and dementia risk; a qualitative study of public attitudes and preferences
}

\author{
Louise Robinson, Claire Dickinson, Eleni Magklara, Lisa Newton, Laura Prato, \\ Claire Bamford
}

To cite: Robinson L, Dickinson C, Magklara E, et al. Proactive approaches to identifying dementia and dementia risk; a qualitative study of public attitudes and preferences. BMJ Open 2018;8:e018677. doi:10.1136/ bmjopen-2017-018677

- Prepublication history and additional material for this paper are available online. To view these files, please visit the journal online (http://dx.doi. org/10.1136/bmjopen-2017018677).

Received 18 July 2017 Revised 7 December 2017 Accepted 13 December 2017

Check for updates

Institute of Health and Society, Newcastle University, Newcastle upon Tyne, UK

Correspondence to

Dr Louise Robinson;

a.l.robinson@newcastle.ac.uk

\section{ABSTRACT}

Objectives The aim of this study was to critically explore the views of the public about the acceptability and feasibility of proactive approaches to earlier dementia diagnosis and also identification of people at high risk of dementia.

Design Qualitative study using task group methodology and thematic data analysis.

Setting Task groups were held either at the university $(n=5)$ or at a carers' centre $(n=1)$. Participants: A convenience sample of 31 of 54 participants identified by local non-statutory agencies took part in a task group. All were aged between 40 years and 80 years, 21 were women and 10 men participated.

Results Despite the use of task group methodology, participants expressed limited understandings of dementia and confusion between proactive approaches. Nevertheless, they highlighted a range of potential benefits and limitations of proactive approaches and the ethical issues raised. There was a preference to embed risk assessment within routine health checks, which focused on achieving a healthier lifestyle, rather than specifically on dementia. Participants emphasised the need to ensure informed consent prior to use of proactive approaches and to provide appropriate support. They also suggested alternative approaches that could potentially facilitate the early detection of dementia or reduce risk at a population level.

Conclusions As international policy on dementia shifts towards a prevention agenda there is growing interest in identifying those at risk of developing dementia. This study provides useful insights into the acceptability of the use of such proactive approaches among the public. The introduction of proactive approaches to dementia identification raises complex practical and ethical issues, particularly in the context of low public understanding of dementia. The importance of better quality information about dementia (and the likelihood of developing dementia) and provision of psychological support for those undergoing risk assessment were highlighted.

\section{INTRODUCTION}

Dementia has a huge impact on people living with the illness and their families and incurs substantial healthcare and societal
Strengths and limitations of this study

- Exploring public views on a clinical area where professionals struggle to achieve consensus poses considerable methodical challenges.

- Due to personnel changes, data analysis was conducted by different researchers from those who undertook data collection; this however facilitated a more critical stance to data interpretation.

- Data collection ceased due to time limitations on the study period; theoretically data collection should have continued until data saturation was achieved.

- Our study explored the perceptions of members of the public, none of whom had dementia; abstract views towards proactive approaches may change if personally facing such assessments.

costs; although more common in older populations, this impact may be greater when dementia occurs earlier in the life course and affects an individual's ability to work and care for their family. ${ }^{1}$ Recent findings from large epidemiological studies have shown considerable inconsistencies in incidence and prevalence trends globally. ${ }^{2-4} \mathrm{~A}$ recent systematic review revealed that in high-income countries the incidence and prevalence of dementia may be declining, probably due to health promotion activities on a wide scale; meanwhile in China and parts of Asia, figures are increasing possibly due to worsening cardiovascular risk profiles. ${ }^{4}$ Although the evidence base around the medical, social and behavioural factors which influence dementia rates is increasing, this is a complex area. ${ }^{3}$ Notwithstanding, increasing evidence that dementia prevalence and incidence may be linked to large-scale, targeted vascular risk reduction and structured chronic illness care for diseases such as diabetes, in addition to increasing age,${ }^{56}$ has led to a shift in global and national policy. ${ }^{78}$ In the absence of a 


\section{Box 1 Task group content and format}

1. Pretask group questionnaire (participant demographic details; personal experience of dementia; knowledge about dementia risk factors).

2. Presentation 1: Dementia assessment and diagnosis

- Introduction to dementia and the process of diagnostic assessment: 'facts and figures'- numbers living with dementia; knowledge to date regarding cause; clinical presentation; dementia subtypes; clinical assessment processes.

Case finding in high-risk groups; factors contributing to dementia; groups at high risk; proactive methods for earlier detection of dementia, for example, case finding.

3. Group discussion 1

4. Presentation 2: Dementia prevention and risk assessment

- Risk assessment-risk factors for dementia.

- Risk assessment tools-general process of disease risk assessment and risk assessment tools; specific dementia risk assessment tools.

Genetic screening and potential future treatments, for example, drugs/vaccines.

5. Group discussion 2

6. Posttask group questionnaire (knowledge about dementia risk factors).

cure, reducing future dementia burden and costs may be best achieved by greater emphasis on prevention which aims to decrease the future number of people developing the illness. ${ }^{7}$ Although general population screening for dementia is not currently advocated, ${ }^{9}$ identifying groups at high risk of developing dementia and giving tailored advice to reduce individual risk, has been recommended by $\mathrm{WHO}^{10}$ as a cost-effective strategy to reduce the global burden of dementia. ${ }^{7}$

This policy shift has resulted in initiatives such as targeted case finding, ${ }^{11}{ }^{12}$ opportunistic assessment to identity possible signs of dementia in patients at high risk, for example, older people aged over 75 years; those with a predisposing medical condition, for example, Parkinson's disease; very high vascular risk in middle age and younger adults (ie, post head injury or Down's syndrome). ${ }^{13} 14$ However such schemes have being introduced into practice although with little evidence of effectiveness. In addition this has led to a growing research focus on developing feasible and valid risk assessment tools to determine, and quantify, a person's risk of developing dementia, with the aim of identifying those who may best benefit from early intervention. ${ }^{15}$ Despite the development of new dementia risk assessment tools, ${ }^{16-18}$ there has been limited research evaluating their acceptability to patients, the public and healthcare professionals. ${ }^{19}$ A systematic review of attitudes to population screening for dementia recommended further qualitative research to explore public and healthcare professional attitudes towards proactive approaches to dementia identification in greater depth. ${ }^{20}$ Determining the barriers and facilitators to the use of dementia risk tools in routine practice is as important as ascertaining their validity. ${ }^{16}$ The aim of this study was to critically explore, using qualitative methods, the views of members of the public about the acceptability and feasibility of proactive approaches to earlier diagnosis and identification of people at high risk of dementia.

\section{METHODS}

We anticipated that levels of knowledge about approaches to earlier identification of dementia and risk assessment among the general public would be low and therefore used task group methodology. ${ }^{21}{ }^{22}$ Data collection in task groups is similar to focus group methodology but includes the presentation of evidence and information about the topic under discussion.

The content and format of the task group are summarised in box 1 . The presentations, developed from recent literature reviews, aimed to provide a summary of evidence-based information in a lay format to facilitate informed discussion. A pilot task group was facilitated by CD, LN and LR with staff from Newcastle University with no specific expertise in dementia and refined prior to the main study. A detailed checklist of methods using the consolidated criteria for qualitative research guidelines ${ }^{23}$ is available in online supplementary file 1 .

Participants were recruited from two local non-statutory organisations: (1) Voice North, a forum for patient/ public involvement in research based in the North-East of England (http://www.voicenorth.org/) and (2) Age UK, a national voluntary organisation, with local branches, which provides services and support to older people (http://www.ageuk.org.uk).

The two organisations used different recruitment approaches: Voice North mailed study information to their members while Age UK advertised the study through posters at meetings of family carers. Both organisations sought consent from interested participants to pass their contact details to the research team. Potential participants were sent further study information and then contacted by a researcher who described the study and answered any questions. Participants were assured that participation was voluntary and that they could withdraw at any time. Written informed consent was secured from participants prior to each task group.

\section{Data collection and analysis}

A further five task groups, facilitated by $\mathrm{CD}$ and LN occurred between December 2015 and July 2016, each lasting approximately 2 hours. Four were held at Newcastle University and the fifth at an Age UK carers' group. All task groups, including the pilot, were digitally recorded and transcribed verbatim; transcripts were checked and anonymised.

A thematic approach to analysis was adopted. ${ }^{24}$ Initially individual researchers read and reread one or two transcripts in detail to become familiar with the data. This stage was particularly important since changes of personnel meant that the analysis was conducted by different researchers $(\mathrm{CB}, \mathrm{EM}, \mathrm{LP})$ to those facilitating 


\section{Box 2 Overview of themes and subthemes}

Confusion around dementia and proactive approaches

- Limited understandings of dementia.

- Making sense of proactive approaches.

Views on proactive approaches to dementia

- Perceived benefits and limitations of proactive approaches.

- Psychological consequences of proactive approaches.

Practical issues in enacting proactive approaches to dementia

- Existing and potential new opportunities for implementation.

- Barriers to implementation of proactive approaches.

- Ethical considerations.

Alternatives to proactive approaches to dementia

- Raising awareness of dementia across the life course.

Health promotion: policy and practice initiatives.

the task groups $(\mathrm{CD}, \mathrm{LN})$. The researchers noted areas of interest and potential codes independently and then compared ideas and discussed the data in workshops.

Following discussion of emergent themes, we developed separate coding frames for facilitator presentations

\section{Box 3 Confusion around dementia and proactive}

\section{approaches}

\section{Limited understanding of dementia}

'Now I don't know to what degree you suffer Alzheimer's, can you get milder cases, more severe cases? Do people just amble along with mild cases of dementia?' (FG3, P17, female)

'Then you say 'dementia', what do you mean? I know that's stupid but my partner's got mild cognitive impairment which could be classified as dementia, because he's got memory problems, or it might be mild cognitive impairment, which is it?' (FG2, P5, female)

'Dementia as far as I'm aware, dementia is one form of Alzheimer's disease' (FG2, P6, male)

'I think language is very powerful. You know, when I was younger, people who had memory problems, do you know, they were a bit wandered you know, they were just getting a bit worn out, you know. The language was kind, but, you talk about dementia, dementia, demented, crazy, and that, that, that encourages people to flee from it, to conceal it and not to share it- '(FG2, P10, male)

\section{Making sense of proactive approaches}

'Does it not smack a bit of Big Brother if you're having a register? 'This person is going to get dementia. This person is going to get dementia. This person isn't.' It smacks of Big Brother sort of...' (FG5, P24, male) 'I don't really know what genetic testing amounts to. It was mooted to me once because l've got an eye condition and it might be genetic, that it was possible to have that done. I was advised that it's quite a big process to go through and it might not give you anything clear at the end of the day.' (FG6, P31, male)

'I mean you really can't prove that if you're like say - do more exercise, change your diet and all that, will it stop you getting that?' (FG3, P14, male)

'And then it (health check) came back with a letter, with a big long 'score this for this, and this for that' but it didn't explain what those numbers meant.' (FG1. P4, female) and group discussions to avoid imposing ideas from the presentations onto participant data. These were then applied to further transcripts and discussed collectively in a further data workshop. Once the coding frames had been agreed, they were applied to all transcripts using Nvivo V.11. Output relating to each theme and subtheme was then reviewed and a narrative summary produced independently (by CB, EM, LP). This was thought to be a more effective way of scrutinising codes than simply checking coding or having two researchers code the data. The narratives were then compared and discussed in further data workshops. Finally a combined narrative was produced for each theme which incorporated the insights and perspectives of different researchers. In a final stage, each transcript was reread in conjunction with the narrative to identify any missing data or issues that had not been captured.

Quotations indicate the focus group (numbered FG1 to FG6), unique participant identifier and gender.

\section{RESULTS}

Of the 54 people invited to take part, 31 agreed to participate in a task group; six groups were completed, each with between three and seven participants. The majority of participants were female $(\mathrm{n}=21,68 \%)$. Around half the sample $(\mathrm{n}=15,48 \%)$ was aged between 60 years and 69 years; $13(42 \%)$ were between 40 years and 59 years and $3(10 \%)$ were $70+$ years. Eighteen $(58 \%)$ knew family members or friends with dementia; a small number had personal experience of proactive approaches to identifying dementia.

Four overarching themes were identified, each of which had several subthemes (box 2). A key theme related to confusion around dementia and proactive approaches. Other themes related to views on proactive approaches, how these might be enacted in practice and alternative approaches that could potentially facilitate the early detection of dementia or reduce risk at a population level.

\section{Confusion around dementia and proactive approaches}

Throughout the task groups there was evidence of limited understanding of dementia and difficulties in distinguishing between approaches such as case finding in high-risk groups, risk assessment and genetic screening. We therefore use the generic term 'proactive approaches' for all of these activities and only differentiate between them where participants were clearly discussing a specific approach. Examples of confusion are illustrated in box 3 and described below.

\section{Limited understanding of dementia}

Participants' understanding of dementia varied, even among those with personal experience of the illness. Uncertainty was expressed about the illness trajectory; boundaries between age-related memory decline, mild cognitive impairment and dementia; and the relationship 


\section{Box 4 Views on proactive approaches}

\section{General views on proactive approaches}

'I don't want to know that I might be going to get it. I don't mind being told that, 'If you do this, this and this you're less likely to have it', just as with heart disease or anything like that.' (FG3, P11, female) 'But perhaps you need different ways, different tools, because what we seem to be saying is that different things suit different people.' (FG2, P9, female)

\section{Perceived benefits and limitations of proactive approaches}

'But I think the point here is if you've got high cholesterol there's a blood test that shows that and the doctor can do something about it. If you're going to develop dementia it's no good telling me unless you can cure it, I can do something myself about it or you can help me.' (FG3, P16, male)

'If there's medication which can slow it down, delay it or whatever and I could have that medication I would be silly to not have it.' (FG5, P27, female)

'... with dementia, I think I would like to know so I could get my life ironed out for my kids, because I wouldn't like to leave them in dire straits.' (FG4, P22, female)

'I think an early diagnosis might be quite frightening at the time but I think it's only fair on your family so that they know what's happening for you and for them to prepare themselves as well for what might happen, if they would be prepared to look after you or if they feel you might need to go into care' (FG3, P17, female)

'The majority of people will say, 'It will never happen to me anyway. I don't need that test. I only smoke 20 fags a day and my neighbour smokes 30 so he's far more likely to...' (FG3, P16, male)

'You can choose to ignore it, but you've been given the information and it's your choice what you do with it, whereas at the minute, it's not there.'

(FG6, P30, female)

\section{Psychological consequences of proactive approaches}

'But there was no sign of it (dementia) in my brain at all. Now I think that was fantastic. I now know that there was no sign of it and that's really, really reassuring.' (FG5, P23, female)

'Do I really want to have the worry about me? It may happen. If I had symptoms of it, then like yourself I would go and probably find out. But I don't feel like I've got symptoms of it, so I don't really want to know that I could have it when I haven't got it, when I feel...' (FG5, P28, female)

'I think there are a percentage of people who wouldn't want to know because they're so scared of the diagnosis because they've seen what happens to people. They've seen relatives go into nursing homes and the eventual outcome.' (FG6, P29, female)

'I did some of these cognitive tests on the internet and I got myself really upset and stressed when I thought I wasn't hitting the time allowed. I dropped out of that because I felt under pressure.' (FG3, P17, female)

between dementia and specific subtypes, for example, Alzheimer's disease (box 3).

All task groups discussed the stigma surrounding dementia which was thought to contribute to the lack of understanding of the condition and a reluctance to be open about symptoms. The fear of dementia was linked to the absence of a cure, potential loss of personhood and devastating effects of advanced dementia which were often conveyed through the language used when talking about people with dementia. Nevertheless many participants felt that societal attitudes towards dementia were improving and drew parallels with other once stigmatising conditions, such as cancer.

\section{Making sense of proactive approaches}

Discussion around specific proactive approaches-case finding, risk assessment and genetic screening — revealed that participants often confused the different methods, sometimes attributing consequences to one approach which related to another. For example, while risk reduction behaviour could reduce the number of people with dementia, case finding would have no impact on prevalence. This distinction was not, however, understood by participants. Participants also voiced opinions which suggested a lack of understanding of specific methods. For example, one participant seemed to think that the case finding method would result in the clear identification of individuals who were going to develop dementia in the future, rather than on identifying those with known risk factors (box 3 ).

The role of genes in dementia similarly seemed to be widely misunderstood by participants. While several participants suggested that genetic screening was the most useful of the proactive approaches, their comments seemed to be based on an implicit assumption that a definitive genetic test is available. Few participants seemed to have understood that a deterministic genetic test is currently only relevant to a small number of individuals with Alzheimer's disease. Although this information was included in the presentation, it did not seem to have been understood by participants many of whom perceived risk of dementia to be largely determined by their personal family history. One participant, who had previously discussed genetic screening in relation to another condition, seemed more aware of the nuanced nature of the information, highlighting the value of personal experience in understanding the issues.

There was a widespread tendency for many participants to see the proactive approaches as providing more definitive information than is the case. The uncertainties surrounding proactive approaches were challenging for many participants and the importance of help with interpreting information about risk and how to act on it were stressed.

\section{Views on proactive approaches to dementia}

Despite their confusion about dementia and proactive approaches, several participants expressed generally positive views towards the concept of proactive approaches, such as 'the earlier the diagnosis the better', without articulating any specific benefits of different methods. Some participants valued general information on risk reduction but not detailed information about their personal risk of developing dementia. Only one participant commented that attitudes to proactive approaches in the abstract might change if personally faced with such approaches. In light of varied individual preferences one participant 
suggested that individuals should be provided with a range of options (box 4).

\section{Perceived benefits and limitations of proactive approaches to dementia}

While some participants questioned the value of proactive approaches for a condition for which no cure was available, others valued the possibility of reducing risk and/or accessing disease-slowing treatment (box 4). Early diagnosis and, to a lesser extent, information on risk were also thought to facilitate planning for the future, both for the individual affected and-often more importantly-their families. Participants acknowledged that a significant limitation of proactive approaches was the fact that information did not automatically result in behaviour change. Regardless of whether or not individuals chose to act on risk information, some participants felt that providing such information increased individual choice.

\section{Psychological consequences of proactive approaches}

Views on the psychological consequences of proactive approaches varied. Participants who had undergone either formal or informal assessment for memory problems described their relief on finding that they did not have dementia; others felt that even receiving a diagnosis of dementia could be a relief. In contrast, other participants felt that proactive approaches while they were asymptomatic (eg, genetic screening, risk assessment) could create considerable anxiety (box 4).

Perceptions of dementia were thought to influence the psychological consequences of proactive approaches; participants felt that people who associated dementia with advanced disease would be afraid of finding out that they were at risk or had a diagnosis of dementia. While discussions focused primarily on the psychological impacts of being given information about risk or possible diagnosis of dementia, the potential of proactive approaches to generate stress and anxiety was also highlighted.

\section{Practical issues in enacting proactive approaches}

This theme explores the suggestions made by participants about how proactive approaches might be integrated into practice and the challenges and questions this may raise.

\section{Existing and potential new opportunities for implementation}

The most common suggestion for introducing proactive approaches was to embed them in routine health check-ups, for example, the annual health review of older adults. Since this approach would focus only on people over a certain age, and dementia can occur in younger adults (eg, those with learning disabilities), routine risk assessments for younger adults, especially those at high risk, were also suggested. This was seen as preferable to introducing a new approach focusing exclusively on dementia and thought by participants to 'normalise' the inclusion of potentially anxiety-provoking questions concerning memory loss (box 5). To address rising general practitioner (GP) workload and financial pressures on services, participants suggested that
Box 5 Existing and potential new opportunities for implementation

\section{Existing and potential new opportunities for} implementation

'My doctors have started to do an annual review of people, so on their birthday they call you in, you go and see the practice nurse and they give you an Ministry of Transport annual car test (MOT) certificate, basically. So it could be at that point that if memory things were introduced to that kind of review... That's just a standard thing so everybody understands that they're going to go through that test, or whatever, then I think incorporating in that could help.' (FG3, P11, female)

'So you could have that health check on all those things, the blood pressure and if that regardless of age, so you don't have to hit the 60 box, if that health check put you in those risk factors then there's follow-up and questions asked about memory' (FG4, P20, female)

'You can go into your doctor's surgery and you don't necessarily have to see a doctor now do you? No, you're seeing a practice nurse. It could be at that level that the tests are done and the risks are quoted to you. Then if you felt that you needed to see a doctor to explain it more or to give you better information...' (FG3, P16, male)

'I think rather than saying, 'Oh, go to the GPs.' I haven't been there for 3 years and I don't need to go there now. Things popping up, but things more widespread across the community so that people will come across them more frequently. In what shape or form, I suppose that's debateable' (FG6, P31, male)

\section{Barriers to implementing proactive approaches}

'It's getting appointments to see them, that's the problem. From personal experience, trying to get to see a GP, you just give up.'

(FG6, P31, male)

'They're too busy. You know, you don't get enough time to do anything like that in my GP's surgery. You never, ever get it, because you don't get enough minutes' appointment.' (FG1, P4, female)

'There's none of the continuity like when you were smaller; we had a family doctor who was there for everybody's health for 50 years.' (FG5, P27, female)

'I do tend to try and- well, I do think I tell the truth in these questionnaires. But you may think, you've got a different idea, perhaps, than somebody else. You might under-exaggerate, or over-exaggerate. You think you're telling the truth, but actually...'

(FG1, P1, female)

'People with dementia, in the early stages, will hide the fact that they've got a problem and can sometimes fool quite a lot of people a lot of the time.' (FG3, P11, female)

'It also requires people to have insight into their memory problem and often people just haven't, have they?' (FG2, P9, female)

\section{Ethical considerations}

'As long as it's an informed consent, but, I think, often [patients] will go with what the GP says because they trust their GP so it's a big responsibility.' (FG2, P5, female)

'You're going to get a lot of people who will go to the GP for a completely different reason and the GP might turn round and say, 'Do you think you have a memory problem?' You think, 'Well, I've come about my in-growing toenail. No, I don't. I'm not here about that.' (FG6, P29, female)

'I think you would need counselling (...) yes, but then the whole thing and then it hits you on an emotional level and that's what you need help.' (FG2, P8, female)

'If you are going to put yourself forward to identify your risk of dementia, then you want to know what will happen if you are at high

Continued 


\section{Box 5 Continued}

risk of dementia. What services are out there, what's the support, available and if there's nothing available for you, you might think, 'Well, what's the point of knowing."' (FG2, P9, female)

'There's always going to be a percentage of the population, it's whether it's to do with health, whether it's to do with whatever, who just don't give a $\mathrm{s}^{\star \star \star}$. But also - but that impacts on us, because eventually we're going to have to pay for the people who don't give a $\mathrm{S}^{\star \star *}$.' (FG4, P20, female)

self-completion questionnaires linked to patient records could be used or that other members of the primary care team could be involved. A few participants suggested that alternative venues such as community centres or health buses, would offer more relaxed environments for proactive approaches.

\section{Barriers to implementation of proactive approaches}

Participants identified three main barriers to implementing a proactive approach in primary care: access to GPs; a lack of continuity of care and the perceived reluctance of people with dementia to acknowledge their problems and seek help. Participants highlighted the difficulties in getting appointments with GPs and the limited time available within appointments. While participants felt that discussing concerns about memory problems would be easier with a GP with whom they had an established relationship, many had experienced a lack of continuity of care (box 5).

In addition to these problems, a number of barriers to early diagnosis were identified. Participants suggested that people with dementia or memory problems might be reluctant to seek help and that even those seen by a GP might present themselves in ways which hid their difficulties. Participants acknowledged the tendency to underestimate alcohol or cigarette consumption and thought that similar behaviour would apply to questions about memory. Concerns were also raised about the ability of people with memory problems to recognise their own difficulties. In light of these concerns, there was a general preference for 'objective' tests (ie, genetic or blood tests-which are not currently available) which did not rely on self-reported information.

\section{Ethical considerations}

Participants identified a number of ethical issues relating to proactive approaches. Some felt that seeking consent prior to proactive approaches was essential to enable patients to make informed decisions and prevent distress. Participants felt that time and support were needed to ensure that patients fully understood the purpose, potential outcomes and implications of tests, and did not simply comply with any questions asked by their GP. Particular concerns were raised about the case-finding approach and how this would be integrated into routine consultations (box 5).
Participants stressed the importance of providing appropriate support services (particularly for people with no close family and limited social networks) prior to introducing proactive approaches. One person with experience of genetic screening highlighted the need for emotional support during the process. A final ethical issue raised during the task groups was the possibility of increasing stigma towards individuals who developed dementia (regardless of whether or not they had taken steps to reduce the risk of developing the disease). This was particularly evident during some task groups in which participants spoke pejoratively about individuals who were perceived not to respond 'appropriately' to risk information.

\section{Alternatives to proactive approaches}

In addition to discussing integration of proactive approaches in primary care, participants suggested introducing approaches to target behaviour change at a population level including increasing awareness of dementia and health promotion or policy initiatives to address risk factors.

\section{Raising awareness of dementia across the life course}

Increasing awareness of dementia was seen as key to: reducing stigma; improving integration of people with dementia; and encouraging people to seek help at an earlier stage. Although awareness raising was discussed in all but one of the task groups, the emphasis varied markedly between groups. Awareness raising was generally seen as relevant to the entire population. Existing UK initiatives such as Dementia Friends ${ }^{25}$ and the Prime Minister's Challenge ${ }^{26}$ were viewed positively (box 6).

Initiatives to raise dementia awareness among specific groups including older people and children were discussed by some groups. Some participants felt that including children was essential to effect a societal change, others argued against targeting younger generations either to avoid burdening them with information about dementia or because of perceptions that they were less likely to engage with an illness strongly related to older age.

Having accessible information presented by a credible source was key to awareness raising. One participant emphasised the importance of avoiding 'medical jargon' and presenting information that people could relate to. A range of existing opportunities for disseminating information were suggested including information in GP surgeries, community centres and patient participation groups.

\section{Health promotion: policy and practice initiatives}

A number of aspects of health promotion were discussed by participants including the extent to which dementia-specific advice was needed and the most appropriate age group to target. There was a general consensus that health promotion should not focus specifically on dementia, but prioritise a healthy lifestyle. As with 


\section{Box 6 Alternatives to proactive approaches}

\section{Raising awareness of dementia across the life course}

'I mean, the dementia friends thing (...) if you can get everybody, you can get kids at school to understand how they can best talk to Granny, how they can react to somebody in the street who is wandering or who's talking to themselves, how to be nice to the person, how to support them.' (FG2, P7, female)

'I think acceptance of it, even it was part of primary/secondary, actually getting it in the schools and getting it at that young age to understand that they're not that person. It's just part of a condition they've got, and acceptance.' (FG6, P30, female)

'Having taught in an further education (FE) college and taught health and social care subjects, 16 to 19 year olds think they're going to live forever. When you try to talk to them about smoking and drinking, it's just over the top of their head. I don't know. Even if they've had experience of grandparents with dementia they think, "It's never going to happen to me and I'm going to live forever and who cares what happens to me when I get to 65 ?"' (FG6, P29, female)

\section{Health promotion: policy and practice initiatives}

'There's a general advice there that's not specific to dementia (...) there's a generic thing going on about 'well if you want to keep your heart going, you need to give up smoking'. It's the same things. So maybe we need to actually pull dementia or the risks of dementia into that general health and well-being better than perhaps we're currently doing.' (FG4, P19, male)

'The schools have enough on their plate with directives coming to say, 'You must teach X amount of maths, English and science' and that's push, push, push. They don't leave a lot of time. They've taken less physical education (PE). So schools don't have that facility' (FG3, P12, female)

'Not everybody is going to get that education, not everybody is going to listen to that education. And there's parental influence on it, social influence on it, economic influence on it. So I think you've got sort of - I believe very strongly in personal choice, but I think there's a point where you've got to look at it more on a sort of society level.' (FG4, P18, male)

With healthy eating, at least now they're going to put this sugar tax on but I would put tax on a load of other things like that. (FG3, P14, male) 'The trouble is with these diets, again, you get it in the papers, 'Don't drink tea, because it's got this... Don't drink coffee because it's got that.' And then a few months down the line, 'Oh, tea's good for you. Coffee is good for you.' What do you believe?' (FG4, P22, female)

awareness raising, views differed over whether health promotion campaigns should target children and the role of schools in promoting a healthy lifestyle (box 5).

Support at a government level was clearly relevant to both awareness raising and health promotion. However, concerns over the lack of reach of these types of initiatives, led some participants to suggest that policy changes might be more effective. Taking certain foods or enforcing limits on the food industry were most frequently suggested as ways of 'enforcing' a healthier lifestyle. While the former was welcomed by some, others were concerned about the potential loss of individual freedom. Concerns were expressed about frequently changing advice on healthy diet and lifestyle since this created uncertainties over how to act on such information and potentially undermined the potential value of both health promotion and providing risk information.

\section{DISCUSSION}

Although presentations were embedded within each task group to introduce dementia case finding, dementia risk assessment and genetic screening, these were insufficient to ensure that participants fully understood the key concepts. They were, however, able to comment on the general principles underlying earlier diagnosis and risk assessment for dementia. Earlier diagnosis was generally welcomed by all participants but views varied regarding risk assessment and genetic screening prior to the emergence of symptoms. There was a preference to embed risk assessment within routine health checks, which focused on achieving a healthier lifestyle, rather focusing specifically on dementia. Participants felt that such health checks should be more widely available and provided by a range of health professionals, including nurses. They also emphasised the need to explore preferences and ensure individuals understand what is involved prior to introducing proactive approaches into routine practice. The confusion evident during the task groups confirms the importance of providing accessible information to enable people to make informed decisions. While participants expressed a strong preference for objective measures rather than those relying on self-reported behaviour, this is at odds with the types of risk assessment tools currently available. Although participants also spoke positively about the need for population approaches to promoting healthy lifestyles, some recognised the potential for negative consequences for individuals who did not adopt recommended lifestyle changes.

The confusion among our public participants around dementia as a condition, and approaches to its earlier identification, is unsurprising in an area where professionals themselves struggle to achieve consensus ${ }^{27} 28$ and expert diagnostic classifications change. ${ }^{29}$ However in terms of public attitudes towards earlier diagnosis of dementia, our findings mirror a systematic review which found that both people with and without cognitive impairment wanted to know sooner, rather than later, if they had dementia in order to better prepare for their future..$^{30}$ Notwithstanding one of the key challenges around introducing approaches to the earlier identification of people with dementia, and also those at higher risk, is a continuing professional stigma around using the diagnostic label of dementia and opening saying the D word to patients despite increasing public awareness campaigns. ${ }^{31}$ Variations in international clinical practice around the use of mild cognitive impairment as a diagnostic label further increase professional inconsistencies. ${ }^{32}$

In terms of identifying those at higher risk of developing dementia, it is interesting to note our participants' preference for such approaches to be part of an integrated, holistic approach to maintaining health in $\mathrm{mid} /$ later life rather than dementia-specific initiatives. 
Such results lend support to current research evaluating holistic health and well-being risk appraisal tools. ${ }^{33}$ Earlier qualitative research exploring public knowledge of dementia risk factors and views on risk reduction approaches found reasonably good knowledge of factors which contribute to healthy ageing. ${ }^{19}$ Interestingly fear of developing dementia, and the need to improve public knowledge about dementia, were considered major motivators among participants towards adopting a healthier lifestyle and improved health behaviours. ${ }^{19}$

Our qualitative study had several limitations. Data collection ceased due to time limitations on the study period; theoretically data collection should have continued until data saturation was achieved. This study explored the perceptions of members of the public, none of whom had dementia. The focus was therefore on case finding, risk assessment/reduction and genetic screening as abstract concepts; participants' views may differ when facing such activities at a personal level. The limited public understanding of dementia ${ }^{34}$ was confirmed in the present study. This suggests that future studies may need to consider either focusing on a single proactive approach (eg, genetic screening) to avoid introducing multiple concepts, or that data collection may need to be conducted over a longer period to enable participants to become familiar with the concepts and facilitate informed discussions, for example, through citizens' juries. ${ }^{35}$ Due to personnel changes, data analysis was conducted by researchers who had no previous involvement in the project. However, as the researchers were more distant from the data, this facilitated a more critical stance and the identification of underlying themes indirectly linked with the study objective.

Recent data on the changing incidence and prevalence of dementia internationally ${ }^{4}$ have generated research interest in prevention through controlling risk factors at both individual and population levels. ${ }^{36}$ While interim findings from a randomised controlled trial of a complex, multicomponent intervention (diet, physical exercise, brain training, vascular risk reduction) targeting individual dementia risk have revealed promising results ${ }^{37}$ the search for feasible and valid risk assessment 'tools' to identify those who would benefit most from such interventions is ongoing. ${ }^{15}$ There has however been little exploration of the acceptability of such approaches to patients and the public ${ }^{19}$ especially in dementia where public understanding of the illness is still low. ${ }^{34}$ With electronic vascular disease risk assessment tools such as Q-RISK2, which allow healthcare professionals to quickly calculate an individual's future risk, ${ }^{38}{ }^{39}$ now embedded in routine primary care practice, similar methods have been used to develop a dementia risk tool. ${ }^{16}$ The validity of this tool is currently under study, it will however be equally important to also explore its acceptability to patients and healthcare professionals, especially if considering the possibility of patient self-administered tools in the future. ${ }^{40}$ In terms of the implications for practice, our study shows that the introduction of proactive approaches to dementia identification should also be accompanied by better quality information about dementia (and the likelihood of developing dementia) and psychological support for those undergoing risk assessment as well as addressing important public concerns about the quality and availability of current dementia care.

As international policy shifts from finding a 'cure' for dementia to focus on more efficient ways of future care provision, including reducing numbers with dementia, research opportunities are beginning to address the prevention as well as 'cure and care' agenda. ${ }^{36}{ }^{41}$ Accurate identification of an individual's risk of developing dementia, in order to identify those who can most benefit from appropriate intervention, will be one part of this new agenda; any future research must however also explore the ethical and personal concerns associated with any newly developed approaches to determining an individual's future risk of developing dementia. While the usefulness and efficiency of general lifestyle checks have been questioned, ${ }^{42}$ these may be a more acceptable way of translating dementia risk reduction approaches into usual care. The considerable confusion among our participants around approaches to earlier identification of both people with possible dementia and those at higher risk of developing the illness in the future suggests an urgent need for greater education focused on dementia risk and individual risk reduction.

Contributors $L R$ conceived the study and obtained project funding. CD and LN carried out data collection; CB, EM and LP were responsible for data analysis. All authors contributed to drafting of the paper and approved the final manuscript.

Funding This paper presents independent research funded by the National Institute for Health Research School for Primary Care Research (NIHR SPCR), project number 261. LR is funded by a National Institute for Health Research Professorship (NIHR-RP-011-043).

Disclaimer The views expressed are those of the author(s) and not necessarily those of the NIHR, the National Health Service or the Department of Health.

Competing interests LR reports grants from the National Institute of Health Research Professorship scheme during the conduct of the study.

Patient consent Obtained.

Ethics approval Newcastle University Faculty of Medical Sciences Ethical Approval Committee.

Provenance and peer review Not commissioned; externally peer reviewed. Data sharing statement No additional data are available.

Open Access This is an Open Access article distributed in accordance with the Creative Commons Attribution Non Commercial (CC BY-NC 4.0) license, which permits others to distribute, remix, adapt, build upon this work non-commercially, and license their derivative works on different terms, provided the original work is properly cited and the use is non-commercial. See: http://creativecommons.org/ licenses/by-nc/4.0/

(C) Article author(s) (or their employer(s) unless otherwise stated in the text of the article) 2018. All rights reserved. No commercial use is permitted unless otherwise expressly granted.

\section{REFERENCES}

1. Alzheimer's Disease International. World alzheimer report 2015: the global impact of dementia: an analysis of prevalence, incidence, cost and trends. London: Alzheimer's Disease International, 2015. 
2. Wu YT, Fratiglioni L, Matthews FE, et al. Dementia in western Europe: epidemiological evidence and implications for policy making. Lancet Neurol 2016;15.

3. Larson EB, Langa KM. What's the "take home" from research on dementia trends? PLoS Med 2017;14:e1002236.

4. Prince M, Ali GC, Guerchet M, et al. Recent global trends in the prevalence and incidence of dementia, and survival with dementia. Alzheimers Res Ther 2016;8.23.

5. Matthews FE, Arthur A, Barnes LE, et al. A two-decade comparison of prevalence of dementia in individuals aged 65 years and older from three geographical areas of England: results of the Cognitive Function and Ageing Study I and II. Lancet 2013;382:1405-12.

6. Matthews FE, Stephan BC, Robinson L, et al. A two decade dementia incidence comparison from the Cognitive Function and Ageing Studies I and II. Nat Commun 2016;7:11398.

7. Alzheimer's Disease International. World alzheimer report 2014: dementia and risk redution. London: Alzheimer's Disease International, 2014.

8. Department of Health. Prime Minister's challenge on dementia 2020. London, 2015.

9. UK National Screening Committee. The UK NSC recommendation on screening for dementia. 2015 www.screening.nhs.uk/dementia (accessed 26 Jun 2017)

10. World Health Organization. Draft global action plan on the public health response to dementia. $2016 \mathrm{http}: / /$ www.who.int/mental health/neurology/dementia/action_plan_consultation/en/ (accessed 12 Jul 2017).

11. Wald NJ, Morris JK. What is case-finding? J Med Screen 1996;3:1.

12. McCartney M. "Case finding" in dementia is simply screening with no evidence of benefit. BMJ 2014;349:g4791.

13. NHS England. Commissioning for quality and innovation (CQUIN) 2014/15 guidance. 2014 https://www.england.nhs.uk/wp-content/ uploads/2014/12/sc-cquin-guid.pdf (accessed 28 May 2017).

14. NHS England. Enhanced Service Specification: Facilitating timely diagnosis and support for people with dementia 2015/16. 2015 https://www.england.nhs.uk/commissioning/wp-content/uploads/ sites/12/2015/03/facilitate-tmly-diag-dementia.pdf (accessed 28 May 2017).

15. Tang EY, Harrison SL, Errington L, et al. Current developments in dementia risk prediction modelling: an updated systematic review. PLoS One 2015;10:e0136181.

16. Walters K, Hardoon S, Petersen I, et al. Predicting dementia risk in primary care: development and validation of the Dementia Risk Score using routinely collected data. BMC Med 2016;14:6.

17. Kivipelto M, Ngandu T, Laatikainen T, et al. Risk score for the prediction of dementia risk in 20 years among middle aged people: a longitudinal, population-based study. Lancet Neurol 2006;5:735-41.

18. Barnes DE, Covinsky KE, Whitmer RA, et al. Dementia risk indices: A framework for identifying individuals with a high dementia risk. Alzheimers Dement 2010;6:138-41.

19. Kim S, Sargent-Cox KA, Anstey KJ. A qualitative study of older and middle-aged adults' perception and attitudes towards dementia and dementia risk reduction. J Adv Nurs 2015;71:1694-703.

20. Martin S, Kelly S, Khan A, et al. Attitudes and preferences towards screening for dementia: a systematic review of the literature. BMC Geriatr 2015;15:66.

21. Hersch J, Jansen J, Barratt A, et al. Women's views on overdiagnosis in breast cancer screening: a qualitative study. BMJ 2013;346:f158.

22. Mair F, May C, Murray E, et al. Understanding the implementation and integration of E-Health services. London: NIHR, 2010.
23. Tong A, Sainsbury P, Craig J. Consolidated criteria for reporting qualitative research (COREQ): a 32-item checklist for interviews and focus groups. Int J Qual Health Care 2007;19:349-57.

24. Braun V, Clarke V. Using thematic analysis in psychology. Qual Res Psychol 2006;3:77-101.

25. Alzheimer's Society. Dementia Friends. https://www.dementiafriends. org.uk/ (accessed 26Jun 2017).

26. Department of Health. Prime Minister's Challenge on Dementia 2020 London, UK: Department of Health, 2015.

27. Petersen RC, Caracciolo B, Brayne C, et al. Mild cognitive impairment: a concept in evolution. J Intern Med 2014;275:214-28.

28. Tang EY, Brayne C, Albanese E, et al. Mild cognitive impairment definitions: more evolution than revolution. Neurodegener Dis Manag 2015;5:11-17.

29. Sachdev PS, Blacker D, Blazer DG, et al. Classifying neurocognitive disorders: the DSM-5 approach. Nat Rev Neurol 2014;10:634-42.

30. van den Dungen P, van Kuijk L, van Marwijk H, et al. Preferences regarding disclosure of a diagnosis of dementia: a systematic review. Int Psychogeriatr 2014;26:1603-18.

31. Robinson L, Gemski A, Abley C, et al. The transition to dementia-individual and family experiences of receiving a diagnosis: a review. Int Psychogeriatr 2011;23:1026-43.

32. Whitehouse PJ, George D. Further limits on labels for cognitive impairment: can we diagnose what we don't know? Am J Geriatr Psychiatry 2011;19:984.

33. Walters K, Kharicha K, Goodman C, et al. Promoting independence, health and well-being for older people: a feasibility study of computer-aided health and social risk appraisal system in primary care. BMC Fam Pract 2017;18:47

34. Mitchell S, Ridley SH, Sancho RM, et al. The future of dementia risk reduction research: barriers and solutions. J Public Health 2017;39:e275.

35. Lenaghan Jo CA. Citizens' juries: theory into practice. London: Institute for Public Policy Research, 1997.

36. Anstey KJ, Eramudugolla R, Hosking DE, et al. Bridging the translation gap: from dementia risk assessment to advice on risk reduction. J Prev Alzheimers Dis 2015;2:189-98.

37. Ngandu T, Lehtisalo J, Solomon A, et al. A 2 year multidomain intervention of diet, exercise, cognitive training, and vascular risk monitoring versus control to prevent cognitive decline in at-risk elderly people (FINGER): a randomised controlled trial. Lancet 2015;385:2255-63

38. Hippisley-Cox J, Coupland C, Vinogradova Y, et al. Predicting cardiovascular risk in England and Wales: prospective derivation and validation of QRISK2. BMJ 2008;336:1475-82.

39. Hippisley-Cox J, Coupland C, Vinogradova Y, et al. Performance of the QRISK cardiovascular risk prediction algorithm in an independent UK sample of patients from general practice: a validation study. Heart 2008;94:34-9.

40. Nolan T, Dack C, Pal K, et al. Patient reactions to a web-based cardiovascular risk calculator in type 2 diabetes: a qualitative study in primary care. Br J Gen Pract 2015;65:e152-e160.

41. Birdi R, Stephan BC, Robinson L, et al. Can we influence the epidemiology of dementia? Perspectives from population-based studies. Postgrad Med J 2015;91:651-4.

42. Frost $\mathrm{H}$, Haw S, Frank J. Promoting health and well-being in later life: interventions in primary care and community settings. Edinburgh: Chief Scientist Office, 2011. 